\title{
Féminité textuelle nel Decameron: la novella di Lisabetta da Messina (IV, 5)
}

Risale ormai al 1979 la celebre distinzione operata da Pierre Bec tra féminité génétique, per definire testi di cui l'autore è sicuramente una donna, e féminité textuelle per testi in cui l'io lirico è femminile, ma l'autore è più probabilmente un uomo. ${ }^{1}$ In questo saggio Bec trattava della tradizione trobadorica, piuttosto avara in esempi di féminité textuelle, rispetto a quella in lingua d'oül o quella gallegoportoghese. In linea di massima, infatti, uno sguardo alle tradizioni liriche romanze medievali rivela una maggiore presenza della tipologia della féminité textuelle nelle diverse tradizioni della Penisola iberica, esclusa la Catalogna, e in quella francese, rispetto a quella occitana e italiana. Quanto questo stato di cose sia da attribuire agli accidenti della trasmissione e dunque della ricezione dei testi lirici è difficile dire, ma allo stato attuale sembra ci sia una preferenza per i generi aristocratisants, per usare un'altra distinzione coniata da Bec, rispetto a quelli popularisants, di cui l'io lirico femminile è caratteristico. ${ }^{2}$ Questi gusti parerebbero aver determinato le scelte di chi ha raccolto la lirica trobadorica, e qui penso soprattutto ai grandi canzonieri veneti, che a loro volta hanno influenzato la tradizione italiana.

La lirica italiana delle origini, dunque, è povera della tipologia della chanson de femme, con qualche eccezione per alcuni testi anonimi siciliani e siculo-toscani a partire, ovviamente, dalla canzone di Rinaldo d'Aquino, Giamäi non mi conforto, che per di più potrebbe essere la più antica poesia della Scuola siciliana. ${ }^{3}$

1 P. Bec, “Trobairitz” et chansons de femme. Contribution à la connaissance du lyrisme féminin au moyen âge, in "Cahiers de civilisation médiévale”, 22, 1979, pp. 235-262.

2 Si veda $\mathrm{P}$. Bec, La lyrique française au moyen âge (XII $-X I I I^{\mathrm{e}}$ siècles): contribution à une typologie des genres poétiques médiévaux, 2 vols., Paris, Picard, 1976-77, vol. I, pp. 97-119, repr. in Id., Écrits sur les troubadours et la lyrique médiévale (1961-1991), Caen, Paradigme, 1992, pp. 283-310, da cui si cita, e le precisazioni di E. W. Poe, Medieval Lyric: the "Trouvères", in The Cambridge History of French Literature, ed. W. Burgwinkle - N. Hammond - E. Wilson, Cambridge, Cambridge University Press, 2011, pp. 67-75 (pp. 69-71).

3 Così ormai R. Coluccia, Introduzione a vol. III di I poeti della Scuola siciliana, ed. R. Antonelli C. Di Girolamo - R. Coluccia, 3 vols., Milano, Mondadori, 2008, pp. xvii-xx.

Nota: Questo contributo si inserisce nelle attività del Progetto di ricerca Voces de mujeres medievales: realidad y ficción (siglos XII-XIV) (FFI2014-55628-P), sovvenzionato dal 'Ministerio de Economía y Competividad' spagnolo.

Charmaine Lee, Università di Salerno

Ә Open Access. (c) 2018 Charmaine Lee, published by De Gruyter. (c) BY-NC-ND This work is licensed under the Creative Commons Attribution-NonCommercial-NoDerivatives 4.0 License. https://doi.org/10.1515/9783110596755-033 
La canzone di Rinaldo rivela che ci fu anche un'influenza della tradizione francese su quella italiana, cosa che si scorge inoltre dalla presenza di manoscritti della lirica d'oïl copiati in Italia, nonché dalla citazione delle poesie di Thibaut de Champagne da parte di Dante nel De vulgari eloquentia. ${ }^{4}$ Credo che è ancora alla tradizione francese che bisogna guardare per spiegare, in parte almeno, la genesi dell'innovazione portata nella narrativa italiana da Boccaccio, che per primo adattò la modalità della féminité textuelle al racconto con la sua Fiammetta, che in parole povere potrebbe essere descritta come "un monologue lyrique, à connotations douleureuses, placé dans la bouche d'une femme", per dirla di nuovo con Bec. ${ }^{5}$ Il romanzo era destinato ad influenzare le diverse tradizioni romanze, e basterebbe menzionare la novela sentimental castigliana fino a Menina e Moça di Bernardim Ribeiro. La soverchiante presenza della voce femminile è anche il tratto caratterizzante il Decameron, dedicato, come si è spesso ripetuto, alle graziosissime donne, vaghe e dilicate, bisognose di distrazioni dalla "malinconia, mossa da focoso disio" (Proemio, 8, 11); "il perno di tutto il sistema di senso del Decameron: le donne, la donna", scrive Amedeo Quondam, che non manca inoltre di osservare come dei dieci membri della lieta brigata che racconta le novelle ben sette sono donne. ${ }^{6}$ Qui di seguito analizzerò un caso specifico di féminité textuelle nel capolavoro di Boccaccio: la novella quinta della quarta giornata, nota come la novella di Lisabetta da Messina, che offre una stratificazione particolarmente ricca di voci femminili, per poi fare alcune considerazioni su quanto l'ottimismo di Quondam sulle donne di Boccaccio sia giustificato.

La quarta giornata del Decameron è posta "sotto il reggimento di Filostrato [abbattuto da amore], si ragiona di coloro li cui amori ebbero infelice fine”. La novella, narrata dalla voce femminile di Filomena, racconta di Lisabetta, che vive a Messina con i tre fratelli, che non approvano il suo amore per Lorenzo, loro servitore, e lo uccidono. Lorenzo appare in sogno alla giovane, le rivela l'assassinio e le indica dove è stato sepolto; sicché lei si reca in questo luogo e gli taglia la testa, che riporta a casa e seppellisce in un vaso, una testa appunto, in cui pianta del basilico, che innaffia con le sue lacrime. Vedendola sempre vicina alla pianta, che cresce rigogliosa, i fratelli si chiedono cosa ci sia nel vaso e scoprono la testa di Lorenzo.

4 Cfr. Dante Alighieri, De vulgari eloquentia, ed. E. Fenzi, Roma, Salerno, 2012, I, 9, 3; II, 5, 4; II, 6, 6 ; in quest'ultimo caso viene attribuito erroneamente Ire d'amors qui en mon cuer repaire (RS 171) a Thibaut e non a Gace Brulé. Si veda ancora F. Brugnolo, Ancora su Siciliani e trovieri: Giacomo da Lentini, Rinaldo d'Aquino, Giacomino Pugliese, in "Romance Philology", 65, 2011, pp. 153-72. 5 P. Bec, Trobairitz, cit., p. 300.

6 A. Quondam, Introduzione, in Giovanni Boccaccio, Decameron, ed. A. Quondam - M. Fiorilla G. Alfano, Milano, Rizzoli (BUR), 2013, pp. 5-65 (a p. 24). Cito il testo del Decameron da Giovanni Boccaccio, Decameron, ed. V. Branca, Torino, Einaudi, 1980. 
La riseppelliscono nella terra, e con la paura che si possa scoprire il delitto, scappano da Messina a Napoli, lasciando sola la sorella a morire del suo dolore. Una volta che la storia si è diffusa, viene composta una canzone sulla vicenda.

Nell'economia della quarta giornata, oltre al tema generale dell'amore tragico, un filo rosso unisce la novella di Lisabetta con la prima della giornata, la storia di Ghismonda e Guiscardo, e con la nona con protagonista Guglielmo di Guardastagno. La testa di Lorenzo, staccato dal corpo e posta nel vaso, richiama il cuore di Guiscardo nella coppa, che a sua volta ricorda il cuore mangiato di Guglielmo e tutte e tre provocano la morte della protagonista. ${ }^{7}$ L'amore di Lisabetta è contrastato dai fratelli perché non considerano Lorenzo alla sua altezza, come Tancredi non pensava che Guiscardo fosse degno della figlia Ghismonda. Anzi, come osserva Giancarlo Alfano, anche se i protagonisti delle novelle di questa giornata non sono sempre membri della classe aristocratica, la fine tragica dell'amore dipende quasi sempre da differenze sociali. ${ }^{8}$ La famiglia di Lisabetta, infatti, è una famiglia della classe mercantile e, per Branca, la novella va letta come parte della cosiddetta "epopea mercantile" che sarebbe il Decameron, in cui il comportamento di Lisabetta contrasta con gli affari e il codice morale dei fratelli mercanti. ${ }^{9}$ Personalmente non condivido la definizione del Decameron come "epopea mercantile" e in questo caso il fatto che la famiglia sia di classe borghese mi sembra un'ennesima prova di come Boccaccio combini i diversi elementi nelle sue novelle. La storia di Lisabetta poteva essere ambientata nel mondo senza tempo della narrativa cortese, come gran parte delle novelle della quarta giornata, che "si mostra particolarmente ricca di riferimenti alla precedente letteratura", ${ }^{10}$ come ben illustrato da quel "secondo che raccontano i provenzali”, che introduce la novella IV, 9. Ma è un tratto costante delle novelle di Boccaccio di essere localizzate in luoghi e tempi precisi così da diventare casi particolari e non più casi tipici. ${ }^{11}$

La novella rientra piuttosto tra quelle che si potrebbero definire novelle angioine, che sono numerosissime nel Decameron. L'azione, come si è visto, si svolge tra Messina, dove è ambientata la vicenda, e Napoli dove si rifugiano i fratelli di Lisabetta, i quali sono toscani di San Gimignano. Branca fa presente che

7 Su questi rapporti tra le novelle si veda M. Picone, La ballata di Lisabetta ("Decameron" IV, 5), in "Cuadernos de filología italiana”, $\mathrm{n}^{\circ}$ extraordinario, 2001, pp. 177-191 (pp. 177-178).

8 G. Alfano, Quarta giornata: Scheda introduttiva, in Decameron, ed. Quondam et al., pp. 657-682 (p. 660).

9 V. Branca, Boccaccio medievale, Firenze, Sansoni, 1970, p. 154.

10 G. Alfano, Quarta giornata, cit., p. 659.

11 Adatto il titolo del saggio di H.-J. Neuschäfer, Il caso tipico e il caso particolare: dalla "vida" alla novella, in M. Picone, ed., Il racconto, Bologna, il Mulino, 1985, pp. 299-308. 
c'erano colonie di mercanti provenienti da questa cittadina a Messina in epoca angioina, e che queste avevano aiutato Carlo II durante la guerra del Vespro; si ha inoltre notizie di trasferimenti di alcuni di questi mercanti a Napoli. Allo stesso modo Pisa, città natale di Lorenzo, vantava una grande presenza di mercanti a Messina fin dai tempi normanni e svevi. ${ }^{12}$ Lambientazione nel Regno, dunque, è precisa ed è l'ennesimo esempio, se ce ne fosse bisogno, dell'influenza che ebbe su Boccaccio il suo soggiorno a Napoli negli anni formativi (1327-1340). Chiaramente tale influenza non è limitata alla conoscenza dei luoghi e della storia, ma abbraccia anche la cultura che, nonostante le preferenze di Roberto il Saggio per quella dotta e latina, era ancora principalmente francese. Ciò permise al giovane Boccaccio di venire a contatto con i diversi filoni della letteratura galloromanza, e più in generale con la cultura volgare della corte che, secondo Francesco Sabatini, era promossa principalmente dalle donne, quelle, magari, che figurano nella Caccia di Diana. ${ }^{13}$ La novella di Lisabetta riflette l'esperienza napoletana, non solo per l'ambientazione, ma nei contenuti, che a loro volta richiamano questi ambienti femminili della corte, e offre numerose sfumature di voci di donna a tutti i livelli.

Gli studi sull'architettura del Decameron concordano nel dividere l'opera in diversi livelli: extradiegetico (interventi dell'autore), intradiegetico (interventi della brigata), e diegetico (le novelle), alle quali si può aggiungere quello metadiegetico (le novelle nelle novelle). ${ }^{14}$ Uno sguardo alla novella IV, 5 rivela che la voce femminile caratterizza ognuno di questi livelli. In primo luogo possiamo considerare come metadiegetico, benché sia un inserto poetico nel racconto e non un racconto, la canzone di cui sono citati i primi due versi alla fine della novella: "Qualesso fu lo malo cristiano / che mi furò la grasta", e da cui scaturisce la storia. Questa canzone non è un'invenzione di Boccaccio e anzi è una precisa testimonianza di un tipo di poesia che doveva circolare a Napoli all'epoca. La cosiddetta "canzone del basilico" fa parte di un piccolo corpus di poesie di tipo popolare che proviene dal Regno di Napoli e, sebbene presenti in manoscritti

12 Decameron, ed. Branca, p. 527, n. 1, e R. Coluccia, Scuola poetica siciliana: una scheda, in Id., Storia lingua e filologia della poesia antica. Scuola siciliana, Dante, Firenze, Franco Cesati, 2016, pp. 31-42, a p. 54. Per una riconsiderazione delle novelle del Decameron in chiave angioina e mediterranea si veda il saggio di S. Kinoshita - J. Jacobs, Ports of Call: Boccaccio's Alatiel in the Medieval Mediterranean, in "Journal of Medieval and Early Modern Studies”, 37/1, 2007, pp. 163-195.

13 F. Sabatini, Napoli angioina. Cultura e società, Napoli, ESI, 1975, p. 85.

14 Cfr. F. Fido, Architettura, in Lessico critico decameroniano, ed. R. Bragantini - P. M. Forni, Torino, Bollati Boringhieri, 1995, pp. 13-33; M. Picone, Autore/narratori, Ivi, pp. 34-59 (pp. 36-42); R. Daniels, Boccaccio's Narrators and Audiences, in The Cambridge Companion to Boccaccio, ed. G. Armstrong - R. Daniels - S. J. Milner, Cambridge, Cambridge University Press, 2015, pp. 36-51. 
copiati in toscana, il fatto che i testi vengano definiti napolitane, ciciliane, calavresi indica una provenienza meridionale, come anche alcuni dei tratti linguistici delle poesie. La stessa canzone del basilico, che non viene definita con uno di questi termini nei manoscritti, inizia con un italianizzato, o meglio toscanizzato (e ipometro) "Questo fu lo malo cristiano", rispetto alla forma meridionale qualesso usato da Boccaccio, e meridionali sono anche grasta 'vaso' e malo cristiano 'persona cattiva'. Il manoscritto più antico che conserva la canzone è Firenze, Biblioteca Mediceo-Laurenziana XLII, 38, che risale alla fine del secolo XIV, ma la testimonianza di Boccaccio implica che circolava prima, forse anche già nel 1282 secondo Rosario Coluccia, che ne è anche l'editore. ${ }^{15}$ Da quanto affermato da Boccaccio si apprende che la canzone, "la quale ancora oggi si canta", era, appunto, cantata e circolava dunque oralmente.

Dal punto di vista della tradizione letteraria italiana questo piccolo corpus di poesie è importante perché rivela la persistenza di una tradizione poetica nell'Italia meridionale oltre la presunta fine della Scuola poetica siciliana. Conferma, inoltre, il passaggio di quest'ultima nel Mezzogiorno prima del suo approdo in Toscana, un fatto che sembra avvalorato anche dall'impiego in questi testi di terminologia derivata dai Siciliani. ${ }^{16}$ In più queste poesie sono significative perché si tratta di testi musicati, come suggeriscono del resto le parole di Boccaccio sulla canzone del basilico. Questo non è affatto comune nella tradizione aulica italiana, ma sembrerebbe riflettere una costante nei gusti della corte angioina, che è la preferenza per la poesia per musica e il registro popolareggiante di ispirazione francese. È noto l'episodio del soggiorno alla corte di Carlo I, durante la Guerra del Vespro, di Adam de la Halle, che ha messo in scena e forse composto il Jeu de Robin et Marion a Napoli; l'opera altro non è che una pastorella drammatizzata, che conclude con un invito alla danza. La danza, infatti, era una forma prediletta dalla corte di Carlo, sia quando era conte di Provenza che re di Sicilia, così che si è potuto affermare che un gruppo di dansas occitane, aggiunto in spazi bianchi

15 R. Coluccia, Tradizioni auliche e popolari nella poesia del regno di Napoli in età angioina, in “Medioevo romanzo", 2, 1975, pp. 44-153; pp. 117-121 per la canzone e p. 64 per la data. Si veda anche F. Sabatini, Napoli angioina, cit., pp. 191-97, 289 n. 161 e ancora R. Coluccia, Boccaccio angioino tra centro e periferia del Regno, in Boccaccio e Napoli. Nuovi materiali per la storia culturale di Napoli nel Trecento, ed. G. Alfano, et al., Firenze, Franco Cesati, 2014, pp. 45-70 (pp. 46-59).

16 Si veda R. Coluccia, Introduzione, cit., pp. xx-xxiv; Id., Scuola poetica siciliana, cit., pp. 37-54, e P. Memelsdorff, “Occhi piangete”: note sull'Ars nova a Napoli, in Boccaccio angioino. Materiali per la storia culturale di Napoli nel Trecento, ed. G. Alfano - T. D’Urso - A. Perriccioli Saggese, Bruxelles, Peter Lang, 2012, pp. 368-392 (pp. 380-382). 
del cosiddetto Chansonnier $d u$ Roi, è “di origine quasi certamente napoletana”. ${ }^{17}$ Formalmente queste dansas somigliano al virelai francese, genere sviluppato proprio da Adam de la Halle. ${ }^{18}$ L'interesse per la musica e per la poesia per musica persistette sicuramente fino al regno di Roberto, per il quale "la musica, a quanto pare, era percepita dai contemporanei quale elemento distintivo". ${ }^{19}$ Anche alla sua corte, dunque, continua la tendenza alla commistione di poesia aulica con quella musicale e dal registro popolareggiante, come rivelato appunto dal corpus delle ciciliane e napolitane, dove non sono neanche rari esempi di féminité textuelle, proprio come la canzone del basilico. Boccaccio, attento alla cultura che lo circondava, sembra aver intercettato questo genere e averlo sfruttato nel discorso da e per le donne che porta avanti nel Decameron e in una novella che si presenta anche come un discorso al femminile.

Nel dire questo non mi riferisco chiaramente alla protagonista della novella, che semmai si contraddistingue per il suo silenzio, ma alla sua ispirazione. ${ }^{20}$ A un primo sguardo si potrebbe pensare che la novella funzioni come una razo per la canzone, considerando pure che all'inizio della novella seguente, con la solita tecnica di collegare i racconti fra di loro, si afferma che "assai volte avevano (le donne) quella canzone udita cantare né mai avean potuto, per domandarne, sapere qual si fosse la cagione per che fosse stata fatta” (IV, 6, 2). L'impiego della parola cagione 'causa, ragione, motivo', come razo, potrebbe riferire alla tradizione dei trovatori allo stesso modo che lo farà la novella IV, 9 derivata dalla vida di Guillem de Cabestany. ${ }^{21}$ Cagione, però, è anche raisun e in questo senso evoca i Lais di Maria di Francia, ai quali in effetti io credo che Boccaccio pensasse nel costruire la novella. La materia della novella non stonerebbe nella collezione di

17 Bibliothèque nationale de France, fonds français 844 (Canzoniere francese $\mathrm{M}$ e occitano W); cfr. A. Radaelli, "Dansas” provenzali del XIII secolo. Appunti sul genere ed edizione critica, Firenze, Alinea, 2004, pp. 11, 217-243; S. Asperti, Carlo d'Angiò e i trovatori. Componenti "provenzali" e angioine nella tradizione manoscritta della lirica trobadorica, Ravenna, Longo, 1995, pp. 121-133.

18 S. Asperti, Carlo d’Angiò, cit., p. 132.

19 P. Memelsdorff, “Occhi piangete”, cit., p. 374; si vedano anche pp. 379-386.

20 Cfr. C. Segre I silenzi di Lisabetta, i silenzi di Boccaccio, in Il testo moltiplicato. Lettura di una novella del "Decameron”, ed. M. Lavagetto, Parma, Pratiche, 1983, pp. 75-85; P. Caraffi, Christine de Pizan lettrice di Boccaccio. Lisabetta immobile e in silenzio, in Boccaccio veneto: settecento anni di incroci mediterranei a Venezia, ed. L. Formisano - R. Morosini, Roma, Aracne, 2015, pp. 77-88. Non sarei, invece, d'accordo con Picone (La ballata, cit., p. 179), che paragona Lisabetta a una trobairitz: non è lei che compone la canzone, ma questa appare come una composizione spontanea tra chi viene a conoscenza della vicenda.

21 Così lo interpretano, per esempio, M. Picone, La ballata, cit., e I. Tufano, "Qual esso fu lo malo cristiano". La canzone e la novella di Lisabetta ("Decameron", IV.5), in "Critica del testo", 10/2, 2007, pp. 225-239. 
Maria che esamina l'amore in tutte le sue sfaccettature e in particolare pone l'accento sulla desmezura come causa delle disgrazie in amore. Lisabetta e Lorenzo:

in questo continuando e avendo insieme assai di buon tempo e di piacere, non seppero sì segretamente fare, che una notte, andando Lisabetta là dove Lorenzo dormiva, che il maggior de' fratelli, senza accorgersene ella, non se ne accorgesse (IV, 5, 6).

Non riescono ad usare discrezione e limitarsi, come Ghismonda e Guiscardo in IV, 1, oppure la dama nel lai di Yonec. E infatti Michelangelo Picone, che forse più di tutti ha insistito sulla presenza dei Lais di Maria tra le fonti di Boccaccio, ha visto nella novella di frate Alberto (IV, 2) una riscrittura in chiave parodica proprio di questo lai. Per lui, anzi, la presenza di questa novella comica nella quarta giornata sarebbe giustificata perché il pubblico ne avrebbe riconosciuto la fonte tragica. Altrove suggerisce, credo a ragione, che "non sarebbe forse errato porre l'intera giornata degli amanti tragici sotto la prestigiosa auctoritas di Maria di Francia”. ${ }^{22}$ Oltre alla novella di frate Alberto, Picone scorge allusioni ai lais di Deus amanz e di Chievrefoil in IV, 1 e, in altre giornate, a Fresne per X, 10 e Laüstic in V, 4, dove elemento scatenante della desmezura è la voglia di ascoltare il canto dell'usignolo. Per quanto riguarda quest'ultima novella, Giancarlo Alfano vede un parallelismo tra "IV, 5 (il tragico caso di Elisabetta da Messina) e la V, 4 (il felice caso di Ricciardetto Mainardi con la figlia di Lizio da Valbona)". ${ }^{23}$ Un dittico di novelle in cui una giovane viene colta sul fatto dalla famiglia: in V, 4 il colpevole Ricciardo teme subito di fare la fine di Lorenzo o di Guglielmo Guardastagno: "parve che gli fosse il cuore del corpo strappato" (V, 4, 42); ma qui le ragioni della natura vengono rispettate e non contrastate, come nelle novelle della quarta giornata, e la storia conclude con un borghese matrimonio di utilità. Ricciardo "è gentile uomo e ricco giovane: noi non possiamo aver di lui altro che buon parentado [...] gli converrà che primieramente la sposi, sì che egli si troverà aver messo l'usignuolo nella gabbia sua e non nell'altrui" (V, 4, 38), commenta la madre di Caterina, sottolineando il significato erotico dell'usignolo. La triste storia di Lisabetta, dunque, richiama i lais nel suo intreccio, ma anche, come si diceva, nel suo rapporto con la canzone. Quel commento finale: "Ma poi a certo tempo divenuta questa cosa manifesta a molti, fu alcun che compuose quella canzone la quale ancora oggi si canta” (IV, 5, 23), chiama a mente le conclusioni dei

22 Cfr. M. Picone, Alle fonti del “Decameron": il caso di frate Alberto, in La parola ritrovata, ed. C. Di Girolamo - I. Paccagnella, Palermo, Sellerio, 1982, pp. 99-117, a p. 102; Id., Il "Decameron”, in Manuale di letteratura italiana. Storia per generi e problemi, ed. F. Brioschi - C. Di Girolamo, 4 vols., Torino, Bollati Boringhieri, 1993, vol. I, pp. 625-654 (pp. 643-654).

23 G. Alfano, Scheda dell'opera, in Decameron, ed. A. Quondam et al., cit., pp. 67-86 (a p. 72). 
Lais di Maria, sempre attenta a collegare l'avventura alla composizione del lai, che come si sa implica anche un testo musicale; per esempio in Fresne (vv. 515-518):
Quant l'aventure fu seüe, Coment ele esteit avenue, Le lai del Freisne en unt trové:
Pur la dame l'unt si numé.

['Quando si riseppe tutta la storia, / così come era avvenuta, / ne composero il lai del Frassino: / dal nome della dama l'hanno così chiamato']. ${ }^{24}$

Ormai mi sembra che non si possa negare che Boccaccio avesse una qualche conoscenza dell'opera di Maria, che avrebbe potuto acquisire alla corte angioina, il che apporta un ulteriore tassello a questa novella 'angioina' come ambientazione ma anche come ispirazione. La canzone, l'opera di Maria puntano agli ambienti culturali della corte, ma si pongono ancora come squisitamente femminili con l'assunzione di una voce femminile anche a livello diegetico.

Infine, ma questi sono i livelli più commentati, la féminité textuelle caratterizza il livello intradiegetico perché la novella è raccontata da Filomena e dunque da una voce femminile che adotta una voce femminile (quella di Maria), che crea un racconto su una poesia a voce femminile. Queste voci hanno poi una ricezione femminile al livello intradiegetico ed extradiegetico, perché oltre al fatto che il Decameron si rivolge alle donne, è chiaro che la ricezione della canzone del basilico, insieme alla sua raisun, è tutta femminile, come si capisce dai commenti delle donne della brigata all'inizio della successiva novella IV, 6, citati sopra:

Quella novella che Filomena aveva detta fu alle donne carissima, per ciò che assai volte avevano quella canzone udita cantare né mai avean potuto, per domandarne, sapere qual si fosse la cagione per che fosse stata fatta (IV, 6, 2).

L'implicazione è che la canzone circolasse in ambienti femminili e direi anche in quegli ambiti femminili che Boccaccio aveva conosciuto da giovane. L'osservazione, già menzionata, che la canzone "ancora oggi si canta" evoca di nuovo il contesto napoletano in cui la canzone doveva essere nota. Non sarei tanto sicura qui che Boccaccio pensasse a Firenze e allo specifico delle dame fiorentine della brigata, come afferma invece Coluccia, sulla base della provenienza fiorentina della tradizione manoscritta. ${ }^{25}$ Tutto il mondo creato da e per

24 Maria di Francia, Lais, ed. G. Angeli, Roma, Carocci, 2003, p. 148 (testo di Rychner). 25 R. Coluccia, Boccaccio angioino, cit., p. 53. 
la brigata ha radici nei luoghi frequentati da altre liete brigate nelle opere giovanili di Boccaccio e fino a Fiammetta, che sono quelli napoletani; a queste opere rimandano ancora i nomi del gruppo di narratori. ${ }^{26}$

La consistente stratificazione di voci femminili in questa novella sembrerebbe confermare ciò che Quondam considera "la clamorosa, spudorata nella sua oltranza, dichiarazione che schiera il Decameron dalla parte delle donne" e che "segna tanta parte del percorso di Boccaccio fino al De mulieribus claris". ${ }^{27} \mathrm{Ma}$, appunto, segna parte del suo percorso e già nel Decameron si può additare tutta una serie di novelle le cui fonti sono da cercare nei fabliaux oppure negli exempla, che non sono affatto teneri con le donne e la cui presenza, insieme a tutte le altre novelle, pur dedicate alle donne, serve a plasmare un nuovo modo di raccontare e non a creare un manifesto pro-femminile. Claude Cazalé Bérard mette in guardia dal soffermarsi sulle problematiche particolari e di leggere le novelle in un contesto più ampio, dove narratori e narratrici superano la dicotomia tra filoginia e misoginia e "vanno annoverati tra i savi uomini e le savie donne di un "nobile castello', dove si avvera il sogno umanistico di una riconciliata confabulazione". ${ }^{28}$ La tesi è condivisibile in parte, ma non toglie che la produzione di Boccaccio contiene anche momenti di notevole misoginia, in primis il Corbaccio, un concentrato dei luoghi comuni medievali contro le donne. Il contrasto tra quest'ultimo e il Decameron ha portato la critica in passato ad ricondurre le opere dove le donne sono viste in una luce positiva al periodo giovanile, semmai anche al soggiorno a Napoli, e quelle più tinte di misoginia alla vecchiaia quando avrebbe rinnegato l'opera con tematiche amorose. Tenderei, invece, a concordare con Francesco Bruni, che punta a una lettura dell'opera boccacciana che tenga presente $\mathrm{i}$ modelli culturali a cui si ispira e il pubblico a cui si rivolge. ${ }^{29}$

Queste due linee culturali corrispondono grosso modo a quella clericale e a quella cortese e Boccaccio ne sembra consapevole fin dagli anni napoletani. Scherza su tale dualismo nella sua Epistola napoletana, una lettera scritta all'amico Francesco dei Bardi in dialetto napoletano, la cui prima redazione risale all'incirca al 1339. Per diversi aspetti questo testo anticipa il Decameron in quanto prende la forma di un racconto comico che imita la lingua parlata,

26 Si veda A. Quondam, Introduzione, cit., pp. 23-24.

27 Ivi, pp. 33, 24.

28 Cl. Cazalé Bérard, Filoginia / misoginia, in Lessico critico decameroniano, cit., pp. 116-141 (cit. a p. 141). Si veda anche F. R. Psaki, Voicing Gender in the "Decameron", in The Cambridge Companion to Boccaccio, cit., pp. 101-117, che opta per una maggiore ambiguità di Boccaccio tra i due poli.

29 F. Bruni, Boccaccio. L’invenzione della letteratura mezzana, Bologna, il Mulino, 1990, p. 74. 
inserito in una cornice composta in una prosa toscana più aulica. ${ }^{30}$ Tra i personaggi presenti nel racconto figura lo stesso Boccaccio, che si scinde in due personalità, due immagini ideali: è l'autore della lettera, Jannetta de Parisse (Giovanni da Parigi), ma è anche l'intellettuale, abbate Ja' Boccaccio, che "nìn juorno, nì notte perzì, fa schitto ca scribere", passa tutto il suo tempo a studiare. Il primo sarebbe il Giovanni che apprezzava la cultura cortese, mentre l'altro, l'abbate, avrebbe ammirato piuttosto la cultura clericale e la biblioteca di Roberto d'Angiò. Infatti sembra che era in questa biblioteca che il giovane Boccaccio avesse letto, copiandone parti in quello che è ora chiamato lo Zibaldone laurenziano (Firenze, Biblioteca Medicea Laurenziana, ms. XXIX 8), il Liber de nuptiis, attribuito a Teofrasto da San Girolamo, che lo incorpora nell'Adversus Iovinianum, e la Dissuasio Valerii ad Rufinum philosophum ne uxorem ducat di Walter Map, incorporata poi nel De nugis curialium. ${ }^{31} \mathrm{Si}$ tratta di due dei testi più sfruttati dalla tradizione misogina medievale, ai quali Boccaccio avrebbe fatto ricorso attraverso gli anni ogni volta che gli tornava comodo, nel Corbaccio, ovviamente, ma anche nel Trattatello in laude di Dante nel momento in cui parla del matrimonio di Dante e di come una moglie possa disturbare la vita speculativa e creativa del poeta. ${ }^{32}$ Si costata, insomma, che Boccaccio si rifaceva a queste due anime culturali fin dagli anni di formazione a Napoli; le avrà magari superate nel Decameron, come sostengono Cazalé Bérard e altri, ma non credo che si possa aderire per questo all'affermazione di Quondam secondo la quale

il Decameron compie una scelta che è scandalosa solo per la sua oltranza, perché da tempo la donna è al centro di tante nuove pratiche culturali che connotano le società cortesi, feudali e urbane. ${ }^{33}$

Non sarei così sicura che la donna sia al centro della società cortese, tanto è vero che è un fatto assodato che la formazione dell'ideologia cortese non corrispose a un miglioramento nella posizione giuridica e sociale delle donne. Infatti la nascita di una classe cavalleresca durante i secoli XI-XII, come risultato dell'acquisizione di feudi che sarebbero poi stati ereditati dai soli maschi primogeniti,

30 Per l'edizione del testo, si veda F. Sabatini, "Prospettive sul parlato nella storia linguistica italiana (con una lettura dell'Epistola napoletana del Boccaccio [1983]”, in Id., Italia linguistica delle origini. Saggi editi dal 1956 al 1996, 2 vols., Lecce, Argo, 1996, vol. II, pp. 425-66 (a pp. 436-41). Si veda anche Id., L'“Epistola napoletana”. Esperimento di genere e di modalità narrative, in Boccaccio e Napoli, cit., pp. 13-21.

31 F. Bruni, Boccaccio, cit., p. 64.

32 Si veda l'edizione di P. G. Ricci in Giovanni Boccaccio, Tutte le opere, a cura di V. Branca, vol. II, Milano, Mondadori, 1974, pp. 423-538.

33 A. Quondam, Introduzione, cit., p. 24. 
è collegata a una nuova concezione del matrimonio come trattato negoziato tra famiglie per evitare la dispersione dei beni. Le donne videro limitato il diritto ad ereditare e ricevevano invece una dote da portare al marito in un matrimonio organizzato da padri e fratelli, come avrebbero voluto fare anche i fratelli di Lisabetta. ${ }^{34} \mathrm{E}$ ancora tenendo presente questo stato di cose che R. Howard Bloch, per esempio, sostiene che la lirica dei trovatori è un dialogo fra uomini che di fatto esclude la donna fin dal suo primo esponente, Guglielmo IX d'Aquitania. ${ }^{35}$ Neanche il romanzo cortese comporta in realtà un apprezzamento maggiore della donna e porta avanti "l'idea che l'importanza della donna stia nel suo rapporto con l'uomo, sia come aiutante attivo che come oggetto passivo", una conferma dell'influenza in questo genere della politica matrimoniale dell'aristocrazia feudale, così i personaggi femminili "non sono donne vere, ma figure all'interno di un discorso maschile". ${ }^{36}$ A tal punto che qualcuno vi ha voluto vedere una metafora dello stupro, come illustrato, per esempio, in Yvain, dall'uccisione di Esclados le Ros, guardiano della fonte incantata, e il successivo possesso della sua vedova, Laudine, da parte di Ivano, sotto la maschera dell'amore cortese. ${ }^{37}$

Non è che le cose andassero meglio nella società cortese che tanto affascinava Boccaccio se pensiamo alla sorte della duchessa Maria di Durazzo (1328-1366), sorella della regina Giovanna I, un caso che Boccaccio non poteva ignorare. Per Maria il nonno, re Roberto, pensava a un'unione con un principe reale, ma a quattordici anni si vide più o meno costretta dalla zia Agnès de Périgord a sposarne il figlio Carlo di Durazzo per avvicinare quest'ultimo al trono. Poi, rimasta vedova a 19 anni, dopo l'esecuzione di Carlo per la sua presunta parte nell'assassinio del marito di Giovanna, Andrea d'Ungheria, Maria fu stuprata da Roberto

34 Sulla situazione delle donne all'epoca si vedano S. Stuard, The Dominion of Gender: Women's Fortunes in the High Middle Ages, in Becoming Visible. Women in European History, ed. R. Bridenthal - C. Koonz - S. Stuard, Boston, Houghton Mifflin, 1987, pp. 153-72; M. Aurell i Cardona, La détérioration du statut de la femme aristocratique en Provence ( $X^{\mathrm{e}}-X{ }^{2} I^{\mathrm{e}}$ siècles), in “Le Moyen Age”, 91, 1985, pp. 5-32; G. Duby, Le chevalier, la femme et le prêtre, Paris, Hachette, 1981; Id. Mâle moyen âge, Paris, Flammarion, 1990; C. N. L. Brooke, Il matrimonio nel Medioevo, Bologna, il Mulino, 1991 [orig. ingl. 1989]; D. Herlihy, La famiglia nel Medioevo, Roma-Bari, Laterza, 1994 [orig. ingl. 1985].

35 R. H. Bloch, Medieval Misogyny and the Invention of Western Romantic Love, Chicago, The University of Chicago Press, 1991, p. 156.

36 Cfr. P. Schine-Gold, The Lady and the Virgin. Image, Attitude and Experience in Twelfth-Century France, Chicago, University of Chicago Press, 1985, p. 42 e S. Gaunt, Gender and Genre in Medieval French Literature, Cambridge, Cambridge University Press, 1995, pp. 71-72.

37 Cfr. R. L. Krueger, Women Readers and the Ideology of Gender in Old French Romance, Cambridge, Cambridge University Press, 1993, p. 40, che riprende lo studio di K. Gravdal, Ravishing Maidens: Writing Rape in Medieval French Literature and Law, Philadelphia, University of Pennsylvania Press, 1991. 
del Balzo, con l'aiuto di suo padre, Ugo, conte di Avellino, per costringerla a un nuovo matrimonio (1350) e evitare quello previsto con Luigi il Grande di Ungheria. Maria almeno ebbe la soddisfazione di poter presiedere all'uccisione di Roberto tre anni dopo. Morì nel 1366, prima del terzo marito Filippo di Taranto, principe di Acaia. ${ }^{38}$

Queste vicende, avvenute ai più alti livelli della società cortese napoletana, sembrano confermare che nella realtà l'atteggiamento verso le donne non fosse profondamente modificato: le donne erano pedine da spostare dove volevano gli uomini, "ristrette”, come scrive lo stesso Boccaccio nel Proemio del Decameron, “da' voleri, da' piaceri, da' comandamenti de' padri, delle madri, de' fratelli e de' mariti” (10). Come lo era del resto Lisabetta, vittima del volere dei suoi fratelli poi abbandonata quando si era comportata contro i loro voleri. L'opera che Boccaccio propone alle donne per consolazione abbraccia appieno la finzione cortese, sfruttando a tutti i livelli le possibilità della féminité textuelle.

38 É. G. Léonard, Gli Angioini di Napoli, Varese, dall’Oglio, 1967 [orig. fr. 1954]. La storia è riportata da Matteo Villani: "disse [Ugo del Balzo] che volea ch'ella fosse moglie di Ruberto suo figliuolo, e per forza le fece consumare il matrimonio"; cfr. Croniche di Giovanni, Matteo e Filippo Villani, vol. II, Trieste, Sezione letterario-artistico del Lloyd Austriaco, 1858, Libro I, cap. xcv, pp. 49-50. Istruttivo a questo riguardo è anche la vita di Aldonza de Mendoza raccontata da Marta Cendón Fernández in questo volume: M. Cendón Fernández, La imagen de doña Aldonza de Mendoza: vida y memoria. 